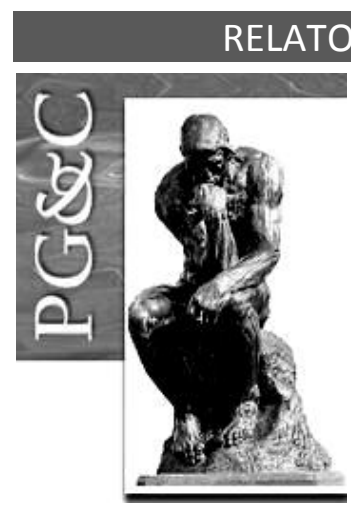

\title{
GRUPOS DE PESQUISA: DINÂMICA DO COMPARTILHAMENTO DE CONHECIMENTO ${ }^{1}$
}

\author{
Alice Munz Fernandes \\ Mestra em Administração pela Universidade de Caxias do Sul, Brasil. \\ E-mail: alicemunz@gmail.com \\ Rejane Remussi \\ Mestra em Administração pela Universidade de Caxias do Sul, Brasil. \\ E-mail: rremussi.br@outlook.com \\ Ana Cristina Fachinelli \\ Doutora em Ciências da Comunicação e da Informação pela Université de \\ Poitiers, França. Professora da Universidade de Caxias do Sul, Brasil. \\ E-mail: afachinelli@gmail.com \\ Fernanda Pauletto D'Arrigo \\ Mestra em Administração pela Universidade de Caxias do Sul, Brasil. \\ E-mail: fernanda.darrigo@yahoo.com.br
}

\begin{abstract}
Resumo
O compartilhamento de conhecimento consiste em um ativo intangível de natureza complexa, configurando-se como um ponto fundamental e delicado, uma vez que está intimamente relacionado à ação, sobretudo em organizações e departamentos intensivos em conhecimento. Entretanto, quando o conhecimento é compartilhado, agrega-se valor às ações, produtos e serviços. Com vistas a isso, esse estudo tem por objetivo examinar a dinâmica do compartilhamento de conhecimento nos grupos de pesquisas, estruturados sob a forma de Núcleos de Pesquisa e Núcleos de Inovação e Desenvolvimento em uma Instituição de Ensino Superior localizada na Serra Gaúcha. Para tanto, a pesquisa é do tipo quantitativa descritiva, operacionalizada por meio de uma survey. Os resultados obtidos demonstram que existe diferença significativa em relação ao compartilhamento do conhecimento explícito, enfatizado sobre a ótica do compartilhamento de informações oficiais, editais e mecanismos de compartilhamento. Observa-se também diferenças entre os grupos em relação ao conhecimento tácito, mais especificamente no compartilhamento das habilidades e lições aprendidas com os erros.
\end{abstract}

Palavras-chave: Conhecimento. Tácito. Explícito. Compartilhamento de Conhecimento.

\section{RESEARCH GROUPS: DYNAMICS OF KNOWLEDGE SHARING}

\section{Abstract}

Knowledge sharing consists of an intangible asset of a complex nature, forming a fundamental and delicate point, since it is closely related to action, especially in knowledge-intensive organizations and departments. However, when knowledge is shared, value is added to actions, products, and services.

\footnotetext{
1 Artigo originado, porém atualizado, do trabalho apresentado sob o título "Compartilhamento de conhecimento tácito e explícito entre grupos de pesquisa" na XV Mostra de Iniciação Científica, PósGraduação, Pesquisa e Extensão do Programa de Pós-Graduação em Administração da Universidade de Caxias do Sul, realizada nos dias 27 e 28 de novembro de 2015 em Caxias do Sul, Rio Grande do Sul, Brasil.
} 
With this in view, this study aims to examine the dynamics of knowledge sharing in research groups, structured in the form of Research Centers and Innovation and Development Centers in a Higher Education Institution located in the Serra Gaúcha. For this, the research is of the descriptive quantitative type, operationalized by means of a survey. The results show that there is a significant difference in relation to the sharing of explicit knowledge, emphasizing the optics of sharing official information, edicts and sharing mechanisms. Differences between groups are also observed in relation to tacit knowledge, more specifically in the sharing of skills and lessons learned from errors.

Keywords: Knowledge. Tacit. Explicit. Knowledge Sharing.

\section{INTRODUÇÃO}

A transição da Era Industrial para a Era do conhecimento modificou tanto os meios de produção, quanto os pensamentos, influenciando nas relações e posições sociais e, deste modo, promovendo vantagem competitiva e agregação de valor (ROSSATTO, 2003). Nesse contexto, o conhecimento torna-se o "fator de produção mais importante na economia da informação" e reside na mente dos indivíduos (SOUTO, 2014, p. 154), impulsionado pela realidade competitiva e globalizada (DAVENPORT; PRUSAK, 1999).

$O$ conhecimento consiste em um recurso cujos atributos não são desvalorizados quando utilizados por diversas pessoas (NONAKA; TOYAMA; HIRATA, 2011), ao contrário, possibilita sua manutenção na organização, promovendo a criação de valor, por meio da maximização da criatividade (GAO; HE; WANG, 2009). Desta forma, configura-se como condição básica para o processo de inovação (STRAUHS; DO, 2012; SOUSA; AMARAL, 2012).

Devido ao fato do conhecimento ser incorporado nos indivíduos, culturas organizacionais, processos e sistemas (BESSANT; TIDD, 2009), é compartilhado nas organizações, quer seja gerenciado ou não (DAVENPORT; PRUSAK, 1999). Desse modo, admite-se que todo sistema social consiste em um mecanismo de produção, reprodução e consequentemente compartilhamento de conhecimento, tratando-se de um sistema epistemológico (ANGELONI, 2002).

Para Strauhs e Do (2012) quando os objetivos são comuns, o compartilhamento de conhecimento estimula o processo de aprendizagem refletindo no beneficiamento de todos os indivíduos envolvidos. Chang et al. (2012) ressaltam que o conhecimento quando compartilhado promove a resolução de problemas e maximiza resultados positivos. Segundo Nonaka e Takeuchi (1997), entende-se como compartilhamento de conhecimento, o comportamento de disseminar o conhecimento adquirido com outros indivíduos, geralmente integrantes da mesma organização. Assim, trata-se de um processo de pessoa a pessoa, configurando-se como um elemento da gestão do conhecimento (RYU; HEEHOO; HAN, 2003).

[...] os indivíduos são importantes. Eles interagem uns com os outros para transcender seus próprios limites e, assim mudar a si próprios. 0 conhecimento subjetivo tácito que alguém possui é externado na forma de conhecimento objetivo explícito, para que seja compartilhado e sintetizado. Assim, os conhecimentos tácitos e explícitos se complementam (NONAKA; TOYAMA; HIRATA, 2011, prefácio).

Entretanto, o conhecimento tácito e explícito não são isolados entre si, mas sim, complementares (NONAKA; TAKEUCHI, 1997). Deste modo, o valor do conhecimento individual se maximiza quando é compartilhado (STYHRE, 2002). Contudo, para que ocorra o compartilhamento de conhecimento é imprescindível a atenção e atitude do indivíduo (YANG, 2010).

Perspectivas em Gestão \& Conhecimento, João Pessoa, v. 8, n. 2, p. 107-125, mai./ago. 2018. 
Historicamente no Brasil, o conhecimento era considerado propriedade exclusiva das organizações de ensino e pesquisa, sobretudo as universidades, todavia, "uma condição de conforto e dormência foi estabelecida com essa suposta exclusividade" (ROCHA, 2004, p. 29). Para Bennet e Bennet (2007, p.146) "o aluno é o envolvido na aquisição de informação e conhecimento relacionados a área de pesquisa, aos resultados da investigação, a aplicação destes resultados na comunidade de públicos de interesse e da miríade de disciplinas e competências necessárias".

Na perspectiva da aprendizagem, o aluno em um grupo de pesquisa vivencia mais que a formação, o ensino e a educação. Ele cria a base do conhecimento profundo, que envolve a reflexão, assimilação, prática, resolução de problemas e a experiência. E assim, integrando a formação, o ensino e aquisição do conhecimento profundo que acontece a mobilização do conhecimento. "A aprendizagem está na base da mobilização do conhecimento - seja ocorrendo em pesquisadores, assistentes de pesquisa, estudantes, profissionais, líderes comunitários, políticos ou outros grupos de interesse" (BENNET; BENNET, 2007, p. 147).

Com vistas a isso, essa pesquisa teve por objetivo examinar a dinâmica do compartilhamento de conhecimento nos Núcleos de Pesquisa (NPs) e Núcleos de Inovação e Desenvolvimento (NIDs) de uma Instituição de Ensino Superior (IES) localizada na Serra Gaúcha. O compartilhamento do conhecimento é identificado como uma das principais áreas da gestão do conhecimento (HENDRIKS, 1999). A relevância do tema deriva particularmente do fato de que ele conecta o conhecimento individual com o conhecimento que reside no nível organizacional tornando-se fonte de valor econômico, competitivo e social. Por outro lado, mesmo que seja reconhecido como um pilar nos esforços de gestão do conhecimento, analisar a dinâmica do compartilhamento pode revelar potenciais escondidos e barreiras críticas que definem as motivações dos sujeitos em acolher o compartilhamento como base de suas práticas profissionais. Tal aspecto torna-se especialmente relevante em contextos intensivos em conhecimento como é o caso do ensino superior.

No que se refere ao ambiente acadêmico, as Instituições de Ensino Superior (IES) concentram o maior número de pesquisadores, cujo compartilhamento de conhecimento relaciona-se com o desenvolvimento da inovação e do desempenho organizacional (MOTTA, 2013). Desse modo, torna-se relevante investigar a forma como ocorre o compartilhamento de conhecimento nesse meio, uma vez que os grupos de pesquisa configuram-se como as organizações formadas por pesquisadores titulados ou em formação que desenvolvem atividades de produção de conhecimento mediante pesquisa (ERDMANN; LANZONI, 2008).

Sendo assim, este estudo é composto além da introdução, por uma revisão da literatura acerca do conceito de conhecimento, seus tipos e formas de compartilhamento. Em seguida, apresenta-se a metodologia de pesquisa, assim como o procedimento de coleta e análise dos dados. Posteriormente, consta a apresentação e discussão dos resultados e, por fim, as considerações finais, abrangendo as limitações do estudo e sugestões para pesquisas futuras.

\section{CONCEITO DE CONHECIMENTO}

O conhecimento está intimamente relacionado com a informação, visto que esta configura-se como meio necessário para construí-lo, extraí-lo (BATESON, 1973) ou/e ainda reestruturá-lo (MACHLUP, 1983). Em relação a isso, Dretske (1981, p.86) corrobora afirmando que o conhecimento é "identificado com a crença produzida ou sustentada pela própria informação". Entretanto, Morin (1986) afirma que o conhecimento se refere à capacidade de compreender as dimensões da realidade, assim como expressá-la de forma continuamente ampla e integral.

Perspectivas em Gestão \& Conhecimento, João Pessoa, v. 8, n. 2, p. 107-125, mai./ago. 2018. 
Conhecimento é uma mistura fluida de experiência condensada, valores, informação contextual e insight experimentado, a qual proporciona uma estrutura para a avaliação e incorporação de novas experiências e informações. Ele tem origem e é aplicado na mente dos conhecedores. Nas organizações, ele costuma estar embutido não só em documentos ou repositórios, mas também em rotinas, processos, práticas e normas organizacionais (DAVENPORT; PRUSAK, 1999, p. 6).

Deste modo, o conhecimento é ao mesmo tempo criado e aplicado na mente do indivíduo, sendo, portanto, dotado de complexidade e imprevisibilidade, estando intimamente relacionado com a ação (DAVEPORT; PRUSSAK, 1999). Segundo Nonaka e Takeuchi (1997, p. 64) conhecimento consiste na crença verdadeira e justificada, configurando-se como um processo dinâmico criado a partir de um fluxo de informação "ancorado nas crenças e compromissos de seu detentor". Para Bessant e Tidd (2009), conhecimento nada mais é do que informação dotada de significado, assim como experiências, princípios e insights tornando-se utilizável e relevante.

$\mathrm{Na}$ filosofia oriental, o conhecimento é definido sob a ótica do racionalismo caracterizando-se como um processo mental perfeito, não apenas como resultado de uma experiência sensorial (NONAKA; TAKEUCHI, 1997). Assim, o conhecimento é acima de tudo, uma questão de como os indivíduos respondem e reagem à realidade (NONAKA; TOYAMA; HIRATA, 2011).

O conhecimento é resultado de um processo cognitivo desencadeado pelos fluxos de novos estímulos, cujo molde ocorre pela necessidade e estoque preliminar de conhecimento já detido pelo indivíduo (ALAVI; DEIDNER, 2001). Para Turban et al. (2010), este origina-se da organização e processamento de dados e informações que promovem a compreensão de determinada situação ou fenômeno, fundamentado na experiência e processo de aprendizagem. Segundo Spender (1996, p. 48) "o conhecimento é um conceito altamente controverso", de modo que "não podemos falar com conhecimento sobre o próprio conhecimento". Nesse sentido, Nonaka e Takeuchi (1997) salientam que o conceito de conhecimento não é definido em termos lógicos.

Todavia, o conhecimento não pode ser confundido com acumulo de informações, visto que trata-se da articulação e legitimação cognitiva destas (ANGELONI, 2002). Nonaka e Takeuchi (1997) definem o conhecimento na dimensão ontológica que se refere ao nível em que este é criado e as condições que a organização ou ambiente oferecem para que os indivíduos criativos gerem e incorporem conhecimentos. Sob a visão de Polanyi (1983), o conhecimento possui uma dimensão epistemológica, baseando-se na distinção entre conhecimento tácito e explicito.

\section{CONHECIMENTO TÁCITO E EXPLÍCITO}

Segundo Polanyi (1983) conhecimento tácito é pessoal, específico ao contexto e de difícil formulação e comunicação, representado pelos modelos mentais individuais e o saber técnico relativo às habilidades inerentes na realização de uma ação. Deste modo, enfatiza que "sabemos mais do que conseguimos dizer" (POLANYI, 1983, p. 24).

Assim, o conhecimento tácito não pode ser totalmente substituído pelo conhecimento explícito, visto que o domínio técnico sobre determinado objeto aprofunda seu conhecimento, no entanto, não é capaz de reproduzir o significado atribuído pelo sujeito (POLANYI, 1983). Trata-se de uma atividade "análoga" (BATESON, 1973), dotada de subjetividade, e, portanto, oriundo da experiência (NONAKA; TAKEUCHI, 1997). Está enraizado na ação e envolvimento do

Perspectivas em Gestão \& Conhecimento, João Pessoa, v. 8, n. 2, p. 107-125, mai./ago. 2018. 
indivíduo com seu contexto, sendo responsável pela forma como este percebe o mundo a sua volta (NONAKA, 2000).

Para Davenport e Prusak (2003), o conhecimento tácito é complexo, desenvolvido e interiorizado pelas pessoas no decorrer de um período de tempo, composto pelo aprendizado acumulado ao longo da vida. Deste modo, "incorpora tanto aprendizado acumulado e enraizado que pode ser impossível separar as regras desse conhecimento do modo de agir do indivíduo", caracterizando-se como ambíguo e de difícil transferência (DAVENPORT; PRUSAK, 1999, p. 86).

Para Angeloni (2002), este conhecimento é composto principalmente pelos elementos contidos na psique do indivíduo, ou seja, abrange habilidades pessoais, sistemas de ideias e, portanto, é difícil de ser gerenciado (STRAUHS; DO, 2012). De um modo geral, o conhecimento tácito consiste no processo de interação, comunicação e coordenação de conhecimento ou de experiência (HASS; HANSEN, 2007), incluindo também o discernimento e instinto individual (ANGELONE, 2008).

Li e Gao (2003) consideram a inserção da consciência subjetiva do indivíduo enquanto base para o desenvolvimento deste conhecimento. Besant e Tidd (2009, p. 216) salientam que o conhecimento tácito "é específico do contexto e difícil de formalizar e comunicar". Por sua vez, Wang e Wang (2012) afirmam que o conhecimento tácito visa melhorar o processo organizacional por meio da troca de informações relevantes, melhores práticas, experiências, lições aprendidas e senso comum reforçando o desempenho da organização (HUANG; WU, 2010). O conhecimento tácito não é distribuído de modo uniforme em uma organização (WANG; WANG; LIANG, 2012), sendo que compartilhá-lo consiste em um desafio organizacional (O'DELL; GRAYSON, 1998).

Em contrapartida, o conhecimento explícito ou codificado refere-se ao conhecimento transmissível em linguagem formal e sistemática (POLANYI, 1983). É de fácil comunicação (DAVENPORT; PRUSAK, 1999), seja mediante especificações de produtos, fórmulas científicas ou programas de computador (NONAKA, 2000). De acordo com Nonaka e Takeuchi (1997), o conhecimento explícito é oriundo da racionalidade, caracterizando-se como metafísico e objetivo. Desse modo, é orientado para uma teoria independente do contexto, ou seja, referese a uma atividade digital (BATESON, 1973). Bessant e Tidd (2009, p. 216) corroboram afirmando que o "conhecimento explícito pode ser expresso em termos numéricos, textuais ou gráficos e, portanto, é mais facilmente comunicado".

A maximização do conhecimento explícito proporciona o aproveitamento do conhecimento formal existente e eleva a experiência de resolução de problemas, refletindo possivelmente na melhoria de processos e produtos (LAWSON et al., 2009). Por sua vez, Wang e Wang (2012), afirmam que o conhecimento explícito facilita a inovação e o desempenho financeiro.

\section{COMPARTILHAMENTO DO CONHECIMENTO}

Segundo Nonaka e Takeuchi (1997, p. 14) "o conhecimento pode ser amplificado ou cristalizado em nível de grupo, através de discussões, compartilhamento de experiências e observações". Entretanto, as organizações possuem bases de dados e redes que armazenam informações, cuja utilização nem sempre é sabida. Nesse sentido, o compartilhamento do conhecimento é comprometido pelo seu próprio grau de codificação (BESSANT; TIDD, 2009).

$\mathrm{O}$ processo de compartilhamento do conhecimento consiste naquele em que a informação de diferentes fontes é partilhada, refletindo na criação de um novo conhecimento (TIDD; BESSANT, 2009). Weggeman (2000) e Van den Hooff, Vijvers e De Ridder (2003) definem duas formas de compartilhamento de conhecimento, quais sejam: doação de conhecimento

Perspectivas em Gestão \& Conhecimento, João Pessoa, v. 8, n. 2, p. 107-125, mai./ago. 2018. 
por meio da comunicação a outros indivíduos e coleta de conhecimento utilizando-se de programas de consultoria que direcionam o compartilhamento.

De acordo com Stewart (1998) a prática do compartilhamento de conhecimento é imprescindível, visto que é inútil possuir conhecimentos relevantes se estes não são compartilhados, estabelecendo-se um fluxo contínuo de conhecimento. Assim, o compartilhamento do conhecimento corresponde a uma dimensão do próprio conhecimento (YAN; CHAN, 2015).

Rossatto (2003) cita que para que haja o compartilhamento do conhecimento é necessária principalmente à interação entre as pessoas, a captura contínua dos conhecimentos alheios, a transmissão de conhecimento por meio de práticas, a participação em eventos, equipes interdisciplinares e valorização de opiniões e ideias. Davenport e Prusak (1999) reconhecem a dificuldade de comunicação e compartilhamento do conhecimento tácito, todavia salientam que mediante narrativas este processo é facilmente viabilizado. "Como regra geral, porém, quanto mais rico e tácito for o conhecimento, mais tecnologia deverá ser usada para possibilitar às pessoas compartilhar aquele conhecimento diretamente" (DAVENPORT; PRUSAK, 1999, p. 117).

Nesse mesmo aspecto, considerando o compartilhamento do conhecimento tácito, Popadiuk e Santos (2010) salientam que há procedimentos informais que facilitam sua partilha, tais como estórias e protocolos não escritos. Ressaltam também que, em contrapartida, sua explicitação ocorre baseada em mapas de conhecimento por meio de banco de dados e intranets.

Indivíduos com capacidade de aprimoramento do conhecimento tácito promovem o compartilhamento deste, pois entendem que tal ação estimula o desenvolvimento não apenas da organização, como também seu próprio universo, consistindo em um círculo virtuoso (STRAUHS; DO, 2012). Segundo Nonaka e Takeuchi (1997), para que o compartilhamento ocorra é imprescindível à existência de sincronia corporal e mental dos indivíduos.

Indo mais além, Bakker et al., (2006) afirma que quando há confiança no relacionamento, os indivíduos maximizam a disponibilidade de compartilhar conhecimento tácito, o que se justifica pelo aumento natural da comunicação (WILLEM; SCARBROUGH, 2006). Para Leana e Pil (2006) indivíduos com modelos mentais semelhantes são mais propensos a compartilhar conhecimento do que aqueles que divergem nesse sentido. Todavia, o compartilhamento de conhecimento em nível grupal estimula a criatividade, a compreensão mútua e promove a obtenção de insights (GONG et al., 2013) e ideias fundamentados no conhecimento de outrem (HUANG; HSIEH; HE, 2014).

O modelo de Gestão de Conhecimento elaborado por Davenport e Prusak (1999) esclarece que para que exista o processo de compartilhamento de conhecimento, é impreterível a adoção de estratégias claras e objetivas, salientando a necessidade de ambientes de trabalho agradáveis que viabilizem a troca de ideias e favoreçam o diálogo (STRAUHS; DO, 2012).

No entanto, para que haja o compartilhamento do conhecimento é necessário à existência de uma habilidade de comunicação adequada associada à utilização de ferramentas cognitivas eficientes (SOUTO, 2014). Todavia, existem alguns fatores que impedem e/ou atrapalham o compartilhamento de conhecimento, quais sejam: falta de confiança mútua, diversidade cultural e de vocabulário, escassez de tempo e de encontros presenciais, existência de status e recompensas aos detentores do conhecimento, falta de capacidade de absorção de conhecimento dos recebedores e intolerância com equívocos e necessidades de ajuda (DAVENPORT; PRUSAK, 1999).

Outro aspecto considerado como condição para a existência do compartilhamento de conhecimento refere-se a modelos relacionais semelhantes, cujos sistemas de incentivos sejam apropriados para o modelo em uso (BOER; BERENDS; BAALEN, 2011). Por sua vez,

Perspectivas em Gestão \& Conhecimento, João Pessoa, v. 8, n. 2, p. 107-125, mai./ago. 2018. 
Stewart (2012) afirma que as pessoas apenas compartilham o que sabem quando percebem claramente o destino de tal conhecimento, enfatizando a existência da intenção do conhecimento.

Pesquisas desenvolvidas por Zheng e Bao (2006) constataram que o compartilhamento do conhecimento relaciona-se positivamente com o comprometimento afetivo da equipe e com a realização de tarefas interdependentes. Segundo Wang, Wang e Liang (2014), o compartilhamento do conhecimento explícito promove a maximização do capital humano e estrutural por meio de troca de documentos, treinamentos e sistemas de tecnologia da informação, por exemplo. No que se refere ao conhecimento tácito, os autores salientam que este impacta diretamente no desempenho financeiro e operacional da organização. Nesse sentido, Santos e Paula (2012) salientam que os estudos concernentes aos distintos aspectos do conhecimento, devem lograr de uma transposição teórico-prática, a fim de que seus benefícios sejam facilmente percebidos.

As pesquisas realizadas por Kim e Yum (2015) apontam que o compartilhamento de conhecimento em si não é capaz de maximizar a eficácia organizacional sem que os destinatários deste conhecimento reconheçam a utilidade e o valor do conhecimento de seus colegas. Para Yeon et al. (2015), as motivações intrínsecas e extrínsecas influenciam na intenção individual de compartilhar conhecimento, predominando o sentimento de prazer em ajudar os outros como impulsionador desse processo. Segundo os autores, as pessoas esperam algum retorno quando compartilham seu conhecimento, seja no âmbito econômico ou sócio emocional.

Diante disso, Macedo et al. (2017, p. 106) corroboram que o "conhecimento com seus novos designs, equipes ágeis e multifuncionais, comunicação multilaterais, informações compartilhadas em redes e comunidades, desafia a gestão das organizações no mundo contemporâneo em que vivemos". No entanto, destaca-se a relação positiva entre desenvolvimento de inovações e a maximização do compartilhamento de informações e conhecimento entre indivíduos em âmbito organizacional, o que enfatiza a relevância de tal temática (SOUSA; AMARAL, 2012).

\section{MÉTODO}

A pesquisa realizada configurou-se, quanto à lógica adotada, como dedutiva, cuja fundamentação teórica foi desenvolvida a priori ao estudo empírico (POPPER, 2003). Em relação à natureza do estudo, tratou-se de uma pesquisa aplicada visto que foi desenvolvida para aplicar suas descobertas em uma problemática específica (COLLIS; HUSSEY, 2005). No que concerne à sua finalidade, caracterizou-se como descritiva, pois visa descrever as características da população ou fenômeno estudado e estabelecer relação entre as variáveis (GIL, 2002).

Quanto à abordagem tratou-se de uma pesquisa quantitativa caracterizada pela objetividade e mensuração dos dados por meio de técnicas estatísticas de análise (BRYMAN, 1988; LAKATOS; MARCONI, 2011). O procedimento técnico utilizado para sua coleta de dados consistiu em um levantamento ou survey com corte transversal, caracterizada por ser lógica, determinística, geral, parcimoniosa e porque "tipicamente examina uma amostra da população" mediante aplicação de um questionário estruturado (BABBIE, 1999, p. 78).

Este estudo adotou como população de pesquisa os integrantes da coordenação, corpo permanente, corpo visitante e corpo técnico dos 17 Núcleos de Pesquisa (NPs) e 16 Núcleos de Inovação e Desenvolvimento (NIDs) de uma IES localizada na Serra Gaúcha, totalizando 206 pesquisadores. Os NPs consistem em grupos de pesquisadores que possuem uma proposta de investigação conjunta desenvolvida através de linhas de pesquisa estabelecidas pela Coordenadoria de Aperfeiçoamento de Pessoal de Nível Superior (CAPES).

Perspectivas em Gestão \& Conhecimento, João Pessoa, v. 8, n. 2, p. 107-125, mai./ago. 2018. 
Por sua vez, os NIDs são grupos de pesquisadores cujas propostas de investigação conjunta de natureza tecnológica, cultural e/ou social, objetivam a geração de novos processos ou produtos intelectuais, assim como novas metodologias e ferramentas científicas para novas aplicações.

Desse modo, adotou-se uma amostragem probabilística do tipo aleatória simples, uma vez que todos os indivíduos que integram a população tiveram as mesmas chances de pertencer a amostra. Assim, considerando a variabilidade dos dados, para um intervalo de confiança de $95 \%$ e um erro padrão de 0,206, a amostra totalizou 73 indivíduos.

$O$ instrumento de coleta de dados consistiu em um questionário estruturado desenvolvido a partir de estudos já validados, sendo que para as variáveis relacionadas ao compartilhamento de conhecimento tácito e explícito foi utilizada a escala de Wang, Wang e Liang (2014) que conta com 13 variáveis. Já para as questões relacionadas ao compartilhamento de conhecimento entre os grupos de pesquisa, utilizou-se a escala desenvolvida por Zheng e Bao (2006) composta por 7 variáveis. Assim, o instrumento final de coleta de dados contou com 20 variáveis, conforme apresentado no Quadro 1.

Quadro 1 - Variáveis de Pesquisa

\begin{tabular}{|c|c|c|c|}
\hline Construto & Variável & Descrição & Referência \\
\hline \multirow{6}{*}{ 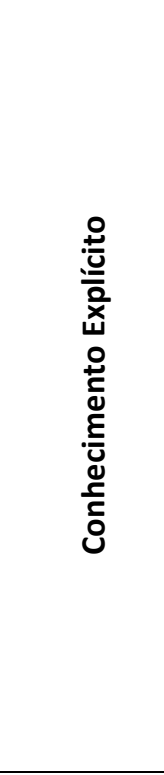 } & EXPLIC1 & $\begin{array}{c}\text { Integrantes do meu grupo de pesquisa frequentemente } \\
\text { compartilham artigos, informações oficiais e editais com os } \\
\text { outros integrantes do grupo. }\end{array}$ & \multirow{6}{*}{$\begin{array}{c}\text { Wang, } \\
\text { Wang e } \\
\text { Liang } \\
\text { (2014) }\end{array}$} \\
\hline & EXPLIC2 & $\begin{array}{c}\text { Integrantes do meu grupo de pesquisa frequentemente } \\
\text { compartilham artigos, informações oficiais e editais por eles } \\
\text { produzidos com os outros integrantes do grupo. }\end{array}$ & \\
\hline & EXPLIC3 & $\begin{array}{l}\text { Integrantes do meu grupo de pesquisa frequentemente } \\
\text { buscam artigos, informações oficiais e editais produzidos } \\
\text { por outros integrantes do grupo em seu trabalho. }\end{array}$ & \\
\hline & EXPLIC4 & $\begin{array}{l}\text { Integrantes do meu grupo de pesquisa frequentemente são } \\
\text { incentivados pelos mecanismos de partilha de } \\
\text { conhecimento (narrativas, manuais, reuniões, etc). }\end{array}$ & \\
\hline & EXPLIC5 & $\begin{array}{l}\text { Para integrantes do meu grupo de pesquisa } \\
\text { frequentemente são oferecidos programas de treinamento } \\
\text { e desenvolvimento. }\end{array}$ & \\
\hline & EXPLIC6 & $\begin{array}{c}\text { O compartilhamento do conhecimento entre os integrantes } \\
\text { do meu grupo de pesquisa é facilitado por sistemas de } \\
\text { Tecnologia da Informação. }\end{array}$ & \\
\hline \multirow{6}{*}{ 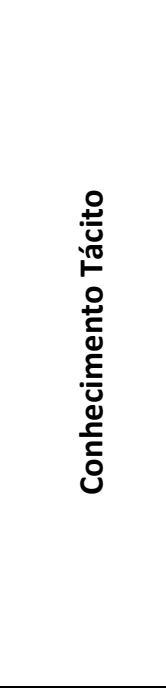 } & TACIT1 & $\begin{array}{l}\text { Integrantes do meu grupo de pesquisa frequentemente } \\
\text { compartilham conhecimento baseados na sua experiência. }\end{array}$ & \multirow{6}{*}{$\begin{array}{l}\text { Wang, } \\
\text { Wang e } \\
\text { Liang } \\
\text { (2014) }\end{array}$} \\
\hline & TACIT2 & $\begin{array}{l}\text { Integrantes do meu grupo de pesquisa frequentemente } \\
\text { buscam conhecimento de outros integrantes baseados na } \\
\text { sua experiência. }\end{array}$ & \\
\hline & TACIT3 & $\begin{array}{l}\text { Integrantes do meu grupo de pesquisa frequentemente } \\
\text { compartilham conhecimento adquiridos com lugares e } \\
\text { pessoas que conheceram com os demais integrantes. }\end{array}$ & \\
\hline & TACIT4 & $\begin{array}{c}\text { Integrantes do meu grupo de pesquisa frequentemente } \\
\text { buscam conhecimento adquiridos com lugares e pessoas } \\
\text { pelos demais integrantes. }\end{array}$ & \\
\hline & TACIT5 & $\begin{array}{l}\text { Integrantes do meu grupo de pesquisa frequentemente } \\
\text { compartilham conhecimento baseado nas suas habilidades. }\end{array}$ & \\
\hline & TACIT6 & $\begin{array}{c}\text { Integrantes do meu grupo de pesquisa frequentemente } \\
\text { buscam conhecimento dos demais integrantes baseadas } \\
\text { nas suas habilidades. }\end{array}$ & \\
\hline
\end{tabular}

Perspectivas em Gestão \& Conhecimento, João Pessoa, v. 8, n. 2, p. 107-125, mai./ago. 2018. 


\begin{tabular}{|c|c|c|c|}
\hline & TACIT7 & $\begin{array}{c}\text { Integrantes do meu grupo de pesquisa frequentemente } \\
\text { compartilham lições dos fracassos passados quando sentem } \\
\text { que é necessário. }\end{array}$ & \\
\hline \multirow{7}{*}{ 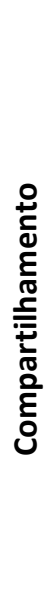 } & COMPAT1 & Quando aprendo algo novo, compartilho com meus colegas. & \multirow{7}{*}{$\begin{array}{l}\text { Zheng e } \\
\text { Bao (2006) }\end{array}$} \\
\hline & COMPAT2 & $\begin{array}{c}\text { Quando meus colegas aprendem algo novo, compartilham } \\
\text { comigo. }\end{array}$ & \\
\hline & COMPAT3 & $\begin{array}{l}\text { O compartilhamento do conhecimento com os meus } \\
\text { colegas é considerado algo normal. }\end{array}$ & \\
\hline & COMPAT4 & $\begin{array}{l}\text { Compartilho a informação que tenho quando meus colegas } \\
\text { me solicitam. }\end{array}$ & \\
\hline & COMPAT5 & $\begin{array}{l}\text { Compartilho minhas habilidades quando solicitado pelos } \\
\text { colegas }\end{array}$ & \\
\hline & COMPAT6 & $\begin{array}{l}\text { Meus colegas compartilham o que sabem quando Ihes } \\
\text { solicito. }\end{array}$ & \\
\hline & COMPAT7 & $\begin{array}{c}\text { Meus colegas compartilham suas habilidades comigo } \\
\text { quando lhes solicito. }\end{array}$ & \\
\hline
\end{tabular}

Fonte: Adaptado de Zheng e Bao (2006) e Wang, Wang e Liang (2014)

Além das variáveis sobre a percepção em relação à dinâmica do compartilhamento de conhecimento no grupo de pesquisa (NID ou NP), o instrumento ainda foi composto por 7 (sete) questões acerca do perfil do respondente da pesquisa, em sua maioria com titulação de mestre na IES. Para tanto, adotou-se uma escala do tipo Likert de 7 pontos considerando o nível de concordância/discordância, sendo "1" para "discordo completamente" e "7" para "concordo completamente".

Ao considerar que todos os trabalhos que originaram as escalas encontravamse originalmente publicados em língua inglesa e foram traduzidos para o português, o questionário adaptado foi submetido à validação de 5 especialistas antes da aplicação. Após a validação, o questionário foi enviado via correio eletrônico (e-mail) para os membros dos grupos de pesquisa. $O$ envio dos mesmos ocorreu em duas etapas de uma semana cada, sendo o primeiro no dia 24 de julho de 2015 , obtendo-se o retorno de 42 respondentes. Um segundo envio do questionário aconteceu no dia 03 de agosto de 2015 onde foi obtido o retorno de mais 31 questionários.

Após a coleta, procedeu-se a validação e análise dos dados. Em um primeiro momento os dados foram analisados por meio da procura de missings ou inconsistências, e então procedeu-se a análise dos dados com o auxílio do Software Statistical Package for the Social Sciences (SPSS) versão 2.0.

Primeiramente foi realizada uma análise descritiva univariada, empregando medidas de variabilidade e de tendência central e posteriormente uma análise multivariada de dados. Para a análise multivariada adotou-se como técnica estatística a One-Way Anova, que permite por meio da comparação das médias de dois ou mais grupos, identificar se as variáveis endógenas em cada categoria do fator são iguais ou não entre si (PESTANA; GAGEIRO, 2005).

\section{ANÁLISE E DISCUSSÃO DOS RESULTADOS}

Quanto à caracterização dos respondentes da pesquisa, os resultados obtidos demonstram que $45,2 \%$ dos respondentes são do sexo masculino e $54,8 \%$ do sexo feminino, cuja faixa etária de maior incidência está entre 37 e 47 anos (41,1\%), seguido por 48 a 58 anos,

Perspectivas em Gestão \& Conhecimento, João Pessoa, v. 8, n. 2, p. 107-125, mai./ago. 2018. 
correspondendo a $35,6 \%$. No que se refere ao grau de escolaridade, constatou-se que $58,9 \%$ possuem doutorado completo, $9,6 \%$ pós-doutorado completo, $24,7 \%$ mestrado, $5,5 \%$ pósdoutorado em andamento e 1,4\% ensino superior.

Quanto à área de formação, identificou-se que $28,8 \%$ dos respondentes são graduados em Ciências Humanas, 17,8\% na área da Saúde e 16,4\% em Ciências Sociais e Aplicadas, sendo que as demais áreas apresentam incidências inferiores.

Em relação ao tipo de grupo de pesquisa ao qual o respondente pertence, observa-se que 45,2\% integram Núcleos de Pesquisa e 45,2\% correspondem aos Núcleos de Inovação e Desenvolvimento. No entanto, constata-se que $9,6 \%$ dos indivíduos são pertencentes a ambos os grupos. O Quadro 2 apresenta a divisão dos grupos de pesquisa a qual os respondentes pertencem.

Quadro 2 - Divisão do grupo de pesquisa

\begin{tabular}{|c|c|}
\hline Divisão do Grupo de Pesquisa & Número de integrantes (\%) \\
\hline Corpo Permanente & $49,3 \%$ \\
\hline Corpo Colaborador & $21,9 \%$ \\
\hline Coordenação & $11 \%$ \\
\hline Corpo Técnico & $5,5 \%$ \\
\hline Mais de um cargo & $12,3 \%$ \\
\hline
\end{tabular}

Fonte: Resultados da pesquisa

Tratando-se do tempo de pertencimento à esses grupos, obteve-se maior incidência na faixa de 2 a 5 anos incompletos (32,9\%), seguido por 5 a 8 anos incompletos, menos de 2 anos $(17,8 \%), 8$ a 11 anos incompletos (11\%) e por fim os indivíduos com mais de 11 anos no grupo de pesquisa $(8,2 \%)$.

A análise descritiva é utilizada para organizar, resumir e descrever os aspectos importantes do comportamento dos dados. Inicialmente realizou-se o teste de KolmogorovSmirnov com o propósito de identificar a normalidade dos dados. O resultado indicou que os dados coletados seguem distribuição normal. Logo após procedeu-se análise descritiva das variáveis por construto.

Foi realizado o cálculo da média, desvio padrão e variância considerando as variáveis que compõem cada construto conforme apresentados na Tabela 1. Desse modo considerando a escala Likert que mensura o grau de concordância/discordância dos respondentes quanto às assertivas, pode-se observar, sob um âmbito geral, a forma como estes percebem a dinâmica de compartilhamento de conhecimento nos seus grupos de pesquisa.

Tabela 1 - Média, desvio padrão e variância

\begin{tabular}{c|c|c|c}
\hline Construto & Média & Desvio Padrão & Variância \\
\hline Conhecimento Tácito & 4,37 & 1,817 & 3,306 \\
\hline Conhecimento Explícito & 4,31 & 1,878 & 3,359 \\
\hline Compartilhamento de conhecimento & 5,39 & 1,59 & 2,562 \\
\hline
\end{tabular}

Fonte: Resultados da pesquisa

Os construtos relacionados ao compartilhamento tácito e explícito apresentam médias próximas (4,37 e 4,31, respectivamente). 0 mesmo acontece para os valores do desvio padrão e da variância. Já para o construto compartilhamento de conhecimento a média correspondeu a 5,39, sendo maior que nas variáveis de conhecimento tácito e explícito. Tais dados indicam que os respondentes atribuíram valores mais altos para as variáveis de compartilhamento de

Perspectivas em Gestão \& Conhecimento, João Pessoa, v. 8, n. 2, p. 107-125, mai./ago. 2018. 
conhecimento, do que as variáveis de conhecimento em si. Tal diferença pode ser relacionada às variáveis que envolvem o construto de compartilhamento de conhecimento, que abrangem "habilidades", "aprendizado" e "sabedoria", que configuram o conhecimento dinâmico.

Para Rabelo et al. (2012), a percepção quanto a relevância de compartilhar conhecimento pode ser entendida como um diferencial competitivo para as organizações, de tosos os segmentos, uma vez que este processo torna-se a base para a aprendizagem dos grupos e da organização sob uma ótica sistêmica. Os autores destacam ainda que este processo dinâmico possibilitará que a organização migre para um novo patamar.

Seguindo os procedimentos de pesquisa, realizou-se a avaliação e confiabilidade da pesquisa por meio do Alpha de Cronbach $(\alpha)$ para verificar a consistência interna das variáveis, cujo valor superior a 0,7 é considerado razoável (PESTANA; GAGEIRO, 2005). Também se calculou o Kaiser-Meyer-Olkin (KMO) que consiste em um indicador estatístico que compara as correlações de ordem zero com correlações parciais existentes entre as variáveis, de modo que 0,7 consiste na média adequada (HAIR et al. 2005). A Tabela 2 apresenta os valores do $\alpha$ e do $\mathrm{KMO}$ considerando os construtos da pesquisa.

Tabela 2 - Alpha de Cronbach e KMO

\begin{tabular}{c|c|c|c}
\hline Construto & $\boldsymbol{\alpha}$ & KMO & Variável \\
\hline Conhecimento Tácito & 0,967 & 0,862 & 7 \\
\hline Conhecimento Explícito & 0,934 & 0,875 & 6 \\
\hline Compartilhamento de conhecimento & 0,912 & 0,771 & 7 \\
\hline
\end{tabular}

Fonte: Resultados da pesquisa

O Alpha de Cronbach foi superior a 0,9 para os construtos, o que garante a consistência interna das variáveis. Já o valor do KMO apresentou como menor resultado 0,771 para o construto de compartilhamento de conhecimento ficando acima do recomendado por Hair et al. (2005). Assim, as medidas de confiabilidade e consistência foram consideradas satisfatórias, então procedeu-se a análise estatística, com o teste Anova.

Através do teste Anova (Tabela 3 ) foi identificada diferença significativa (inferior a 0,005 ) em 4 (quatro) variáveis, das quais 2 (duas) referem-se ao conhecimento explícito (informações oficiais, editais e mecanismos de partilha de conhecimento) e 2 (duas) ao conhecimento tácito (habilidades e lições de fracassos).

\begin{tabular}{|c|c|c|c|c|}
\hline \multicolumn{2}{|c|}{ Variáveis } & \multirow{2}{*}{$\begin{array}{c}\begin{array}{c}\text { Mínimo } \\
\text { Quadrado }\end{array} \\
17,596 \\
\end{array}$} & \multirow{2}{*}{$\begin{array}{c}\mathbf{F} \\
5,626 \\
\end{array}$} & \multirow{2}{*}{$\begin{array}{c}\text { SIG. } \\
0,005\end{array}$} \\
\hline EXР॥1 & Dentre o grupo & & & \\
\hline EXPLII & Entre grupos & 3,127 & & \\
\hline \multirow{2}{*}{ EXPLI2 } & Dentre o grupo & 14,734 & 4,286 & 0,018 \\
\hline & Entre grupos & 3,437 & & \\
\hline \multirow{2}{*}{ EXPLI3 } & Dentre o grupo & 6,899 & 2,425 & 0,096 \\
\hline & Entre grupos & 2,845 & & \\
\hline \multirow{2}{*}{ EXPLI4 } & Dentre o grupo & 20,855 & 6,073 & 0,004 \\
\hline & Entre grupos & 3,434 & & \\
\hline \multirow{2}{*}{ EXPLI5 } & Dentre o grupo & 11,634 & 4,145 & 0,02 \\
\hline & Entre grupos & 2,807 & & \\
\hline \multirow{2}{*}{ EXPLI6 } & Dentre o grupo & 19,988 & 5,591 & 0,006 \\
\hline & Entre grupos & 3,575 & & \\
\hline
\end{tabular}

Perspectivas em Gestão \& Conhecimento, João Pessoa, v. 8, n. 2, p. 107-125, mai./ago. 2018. 
Aline Munz Fernandes et al.

\begin{tabular}{|c|c|c|c|c|}
\hline \multirow{2}{*}{ TACIT1 } & Dentre o grupo & 14,515 & 5,146 & 0,008 \\
\hline & Entre grupos & 2,821 & & \\
\hline \multirow{2}{*}{ TACIT2 } & Dentre o grupo & 13,158 & 4,366 & 0,016 \\
\hline & Entre grupos & 3,013 & & \\
\hline \multirow{2}{*}{ TACIT3 } & Dentre o grupo & 12,989 & 4,137 & 0,02 \\
\hline & Entre grupos & 3,14 & & \\
\hline \multirow{2}{*}{ TACIT4 } & Dentre o grupo & 10,511 & 3,154 & 0,049 \\
\hline & Entre grupos & 3,333 & & \\
\hline \multirow{2}{*}{ TACIT5 } & Dentre o grupo & 15,578 & 5,642 & 0,005 \\
\hline & Entre grupos & 2,761 & & \\
\hline \multirow{2}{*}{ TACIT6 } & Dentre o grupo & 12,247 & 4,434 & 0,015 \\
\hline & Entre grupos & 2,762 & & \\
\hline \multirow{2}{*}{ TACIT7 } & Dentre o grupo & 22,099 & 7,147 & 0,001 \\
\hline & Entre grupos & 3,092 & & \\
\hline \multirow{2}{*}{ COMPART1 } & Dentre o grupo & 11,62 & 3,896 & 0,025 \\
\hline & Entre grupos & 2,983 & & \\
\hline \multirow{2}{*}{ COMPART2 } & Dentre o grupo & 11,492 & 3,769 & 0,028 \\
\hline & Entre grupos & 3,049 & & \\
\hline \multirow{2}{*}{ COMPART3 } & Dentre o grupo & 12,285 & 4,173 & 0,019 \\
\hline & Entre grupos & 2,944 & & \\
\hline \multirow{2}{*}{ COMPART4 } & Dentre o grupo & 2,875 & 1,577 & 0,214 \\
\hline & Entre grupos & 1,823 & & \\
\hline \multirow{2}{*}{ COMPART5 } & Dentre o grupo & 2,025 & 1,053 & 0,354 \\
\hline & Entre grupos & 1,923 & & \\
\hline \multirow{2}{*}{ COMPART6 } & Dentre o grupo & 4,44 & 2,044 & 0,137 \\
\hline & Entre grupos & 2,173 & & \\
\hline \multirow{2}{*}{ COMPART7 } & Dentre o grupo & 4,008 & 1,859 & 0,164 \\
\hline & Entre grupos & 2,156 & & \\
\hline
\end{tabular}

Fonte: Resultados da pesquisa

A Anova demonstra a análise de variância dentre os respondentes e entre os grupos de respondentes. Neste caso, a análise de significância aponta que a variável TACIT7 referente a lições de fracasso, apresentou diferenças significantes dentre os respondentes (sig = 0,001; F= 7,147). A variável EXPLIC4, referente a mecanismos de partilha de conhecimento (narrativas, manuais, reuniões, etc) apresentou a segunda maior diferença significativa entre os respondentes ( $\operatorname{sig}=0,004 ; F=6,073$ ). As variáveis TACIT 5, referente a habilidades e EXPLICIT1 referente a artigos, informações oficiais e editais também apresentaram diferenças significativas ao nível de 0,005 ( $F=5,642$ e $F=5,626$ respectivamente).

Mesmo que a Anova demonstre a variação entre os grupos de respondentes, não é possível identificar entre quais grupos que as diferenças significativas ocorreram. Para atender a esta demanda foi realizado o Teste de Tukey entre as variáveis com diferenças significativas apontados pela Anova, conforme apresentado na Tabela 4.

Tabela 4 - Teste Tukey

\begin{tabular}{l|l|l|c|c}
\hline \multicolumn{1}{c|}{ Variáveis } & \multicolumn{2}{|c|}{ Grupos } & Erro & Sig. \\
\hline EXPLI1 & NP & NID & 0,435 & 0,005 \\
& & NP/NID & 0,736 & 0,221 \\
\cline { 2 - 5 } & NID & NP & 0,435 & 0,005 \\
& & NP/NID & 0,736 & 0,964 \\
\hline
\end{tabular}

Perspectivas em Gestão \& Conhecimento, João Pessoa, v. 8, n. 2, p. 107-125, mai./ago. 2018. 


\begin{tabular}{|c|c|c|c|c|}
\hline & NP/NID & $\begin{array}{l}\text { NP } \\
\text { NID }\end{array}$ & $\begin{array}{l}0,736 \\
0,736\end{array}$ & $\begin{array}{l}0,221 \\
0,964\end{array}$ \\
\hline \multirow[t]{3}{*}{ EXPLI4 } & NP & $\begin{array}{l}\text { NID } \\
\text { NP/NID }\end{array}$ & $\begin{array}{l}0,456 \\
0,771\end{array}$ & $\begin{array}{l}0,003 \\
0,314\end{array}$ \\
\hline & NID & $\begin{array}{l}\text { NP } \\
\text { NP/NID }\end{array}$ & $\begin{array}{l}0,456 \\
0,771\end{array}$ & $\begin{array}{l}0,003 \\
0,832\end{array}$ \\
\hline & NP/NID & $\begin{array}{l}\text { NP } \\
\text { NID }\end{array}$ & $\begin{array}{l}0,771 \\
0,771\end{array}$ & $\begin{array}{l}0,314 \\
0,832\end{array}$ \\
\hline \multirow[t]{3}{*}{ TACIT5 } & NP & $\begin{array}{l}\text { NID } \\
\text { NP/NID }\end{array}$ & $\begin{array}{l}0,409 \\
0,691\end{array}$ & $\begin{array}{l}0,005 \\
0,198\end{array}$ \\
\hline & NID & $\begin{array}{l}\text { NP } \\
\text { NP/NID }\end{array}$ & $\begin{array}{l}0,409 \\
0,691\end{array}$ & $\begin{array}{l}0,005 \\
0,981\end{array}$ \\
\hline & NP/NID & $\begin{array}{l}\text { NP } \\
\text { NID }\end{array}$ & $\begin{array}{l}0,691 \\
0,691\end{array}$ & $\begin{array}{l}0,198 \\
0,981\end{array}$ \\
\hline \multirow[t]{3}{*}{ TACIT7 } & NP & $\begin{array}{l}\text { NID } \\
\text { NP/NID }\end{array}$ & $\begin{array}{l}0,433 \\
0,732\end{array}$ & $\begin{array}{l}0,001 \\
0,994\end{array}$ \\
\hline & NID & $\begin{array}{l}\text { NP } \\
\text { NP/NID }\end{array}$ & $\begin{array}{l}0,433 \\
0,732\end{array}$ & $\begin{array}{l}0,001 \\
0,107\end{array}$ \\
\hline & NP/NID & $\begin{array}{l}\text { NP } \\
\text { NID }\end{array}$ & $\begin{array}{l}0,732 \\
0,732\end{array}$ & $\begin{array}{l}0,994 \\
0,107\end{array}$ \\
\hline
\end{tabular}

Fonte: Resultados da pesquisa

Percebe-se que em todas as variáveis as diferenças significativas nas respostas se dão nos respondentes associados a apenas um grupo de pesquisa (NP ou NID). As respostas de membros que fazem parte tanto de NP como de NID não apresentaram diferenças significativas entre os integrantes de apenas um grupo.

Ao analisar as questões, percebe-se que a diferença mais significante $(0,001)$ entre o grupo NP e os NID corresponde a percepção que os integrantes têm sobre o compartilhamento dos fracassos e erros com a equipe (TACIT7). Ainda no campo do conhecimento tácito, os grupos também apresentaram diferenças significativas a respeito do compartilhamento das habilidades dos integrantes do grupo de pesquisa (TACIT 5).

Nesse sentido, Rabelo et al. (2012) corroboram que para que o compartilhamento do conhecimento seja algo motivador, é necessário considerar as complexidades humanas, haja vista que os limites nesse processo são advindos das crenças e suposições pessoais. Logo, evidencia-se a importância de se trabalhar com tais elementos inerentes a natureza humana. Outro elemento que pode justificar o motivo pelo qual as falhas e erros não são compartilhados consiste no próprio instinto de competição, moldado sob a égide da produção e da proteção pessoal (PMBOK, 2008).

Em relação às questões do conhecimento explícito, os grupos apresentaram diferenças significativas em relação aos incentivos ao compartilhamento do conhecimento (EXPLIC4). Os grupos também apresentaram diferenças significativas no compartilhamento de artigos, informações oficiais e editais com os outros integrantes do grupo (EXPLIC1).

Freitas Júnior et al. (2017), propuseram um modelo de Sistema de Gestão do Conhecimento, cuja fundamentação basilar aponta que o aprendizado colaborativo e cooperativo em rede configura-se como uma forma de incrementar o potencial de inteligência coletiva dos pesquisadores. Assim, o compartilhamento do conhecimento, enquanto elemento

Perspectivas em Gestão \& Conhecimento, João Pessoa, v. 8, n. 2, p. 107-125, mai./ago. 2018. 
da gestão do conhecimento, ocorreria de modo organizado e sistematizado, possibilitando ganhos em maiores escalas.

\section{CONSIDERAÇÕES FINAIS}

O objetivo principal do estudo foi examinar a dinâmica do compartilhamento de conhecimento nos Núcleos de Pesquisa (NPs) e Núcleos de Inovação e Desenvolvimento (NIDs) de uma Instituição de Ensino Superior (IES) localizada na Serra Gaúcha. Isso porque, ao considerar o papel dos grupos de pesquisa das IES na produção do conhecimento científico e no avanço da ciência, estudar a dinâmica do conhecimento entre os grupos de pesquisa pode resultar em achados relevantes para o ensino e a pesquisa.

O conhecimento criado no grupo de pesquisa, e compartilhado com os demais grupos, bem como a comunidade, favorece o desenvolvimento da inovação e do desempenho organizacional (MOTTA, 2013). Por isso, conclui-se que o compartilhamento de conhecimento entre os grupos de pesquisa torna-se um elemento fundamental para que o conhecimento criado em um grupo de pesquisa seja disseminado a sociedade. Desse modo, pode amplificar ou cristalizar o conhecimento por meio de discussões, compartilhamento de experiências e observações, ao encontro do que é defendido por Nonaka e Takeuchi (1997).

Nos achados da pesquisa, identificou-se que os grupos de pesquisa da IES estudada, apresentam diferentes percepções em relação ao compartilhamento de conhecimento tácito e explícito dentro dos grupos. Ao tratar o conhecimento explícito, a principal variação de percepções está relacionada às ações de compartilhamento de artigos, informações oficiais e editais com os outros integrantes do grupo e aos incentivos ao compartilhamento de conhecimento. Ou seja, os membros dos grupos não concordaram com as afirmações que demais membros do grupo compartilham materiais escritos (artigos, informações oficiais e editais) e nem recebem incentivo para isto.

Esta diferença pode ser resultados da cultura de compartilhamento existente nos grupos. A cultura de compartilhamento cria um espaço que favorece a mobilização do conhecimento, o que leva a aprendizagem e a construção do conhecimento profundo (BENNET; BENNET, 2007). Quando a cultura do grupo não estimula a interação, a tendência é que o compartilhamento de conhecimento não aconteça, dificultando a criação de novos conhecimentos.

Quanto ao conhecimento tácito, as principais variações envolveram as ações dos membros do grupo de pesquisa em compartilhar conhecimento baseado nas habilidades, e com as lições aprendidas por fracassos passados. Compartilhar as lições aprendidas pelos fracassos é uma importante forma de aprendizado, pois evita que os demais membros do grupo gastem tempo e recursos em vão em tentativas que já não foram bem-sucedidas. Quando os membros compartilham os insucessos, podem dedicar esforços a encontrar novas soluções para alcançar o sucesso.

Destaca-se que não foram observadas diferenças significativas entre os participantes de ambos os grupos. Ou seja, as diferenças percebidas em relação ao compartilhamento de conhecimento tácito e explicito dentro dos grupos de pesquisa é identificado apenas por membros que participam de apenas um grupo de pesquisa. Esta percepção pode ser relacionada os processos e mecanismos (formais e informais) adotados pelos grupos para $o$ compartilhamento de conhecimento.

Por isso, sugere-se como pesquisas futuras a realização de estudos que analisem em profundidade processos formais e informais do compartilhamento de conhecimento nos grupos e entre os grupos de pesquisa. Tal pesquisa pode identificar quais aspectos favorecem ou impedem o desenvolvimento de uma cultura de compartilhamento de conhecimento entre os grupos de pesquisa.

Perspectivas em Gestão \& Conhecimento, João Pessoa, v. 8, n. 2, p. 107-125, mai./ago. 2018. 
Reconhecem-se as limitações do estudo quanto a não validação trans(cultural) do instrumento de coleta de dados utilizado, haja vista que apesar de ser validado por especialistas, não foi testado empiricamente no contexto nacional em investigações anteriores. Também aponta-se como limitação a análise apenas da percepção dos respondentes a respeito do compartilhamento do conhecimento, não utilizando nenhum outro indicador para comparar a efetividade de compartilhamento do conhecimento.

Assim, como perspectiva de estudos futuros, a realização de uma comparação dos resultados do compartilhamento de conhecimento tácito e explícito dos grupos estudados com indicadores de desempenho complementaria a investigação realizada. Analisando os indicadores de desempenho dos grupos, pode-se analisar a correlação entre 0 compartilhamento de conhecimento e os resultados do grupo.

\section{REFERÊNCIAS}

ALAVI, M.; LEIDNER, D. E. Review: knowledge management and knowledge management systems: conceptual foundations and research issues. MIS Quartely, v. 25, n. 1, p. 107-136, 2001.

ANGELONE, M. T. Organizações do conhecimento: infraestrutura, pessoas e tecnologia. São Paulo: Saraiva, 2008.

ANGELONI, M. T. (Coord). Organizações do conhecimento: infra-estrutura, pessoas e tecnologias. São Paulo: Saraiva, 2002.

BABBIE, E. Métodos de pesquisas de survey. Belo Horizonte: UFMG, 1999.

BAKKER, M.; LEENDERS, R.; GABBAY, S.M.; KRATZER, J.; VAN ENGELEN,J. M. L. Is trust really social capital? Knowledge sharing in product development projects. The Learning Organization, v.13, p. 594-605, 2006.

BATESON, G. Steps to an Ecology of Mind. Londres: Paladin, 1973.

BENNET, D.; BENNET, A. Knowledge mobilization in the social sciences and humanities: Moving from Research to Action. Mqi Press, 2007.

BESSANT, J.; TIDD, J. Inovação e empreendedorismo. Porto Alegre: Bookman, 2009.

BOER, N. I.; BERENDS, H.; BAALEN, P. V. Relational models for knowledge sharing behavior. European Management Journal, v. 29, p. 85- 97, 2011.

BRYMAN, A. Quantity and quality in social research. London: Routledge, 1988.

CHANG, C.W.; HUANG, C.W. H.C.; CHIANG, C.Y.; HSU C, P.; CHANG, C.C. Social capital and knowledge sharing: effects on patient safety. Journal of Advanced Nursing, v. 68, n.8, p. 17931803, 2012.

COLLIS, J.; HUSSEY, R. Pesquisa em administração: um guia prático para alunos de graduação e pós-graduação. 2. ed. Porto Alegre: Bookman, 2005.

Perspectivas em Gestão \& Conhecimento, João Pessoa, v. 8, n. 2, p. 107-125, mai./ago. 2018. 
DAVENPORT, T.; PRUSAK, L. Conhecimento empresarial. Rio de Janeiro: Campus, 1999.

Conhecimento empresarial: como as organizações gerenciam seu capital intelectual. Rio de Janeiro: Campus, 2003.

ERDMANN, A. L.; LANZONI, G. M. M. Características dos grupos de pesquisa da enfermagem brasileira certificados pelo CNPQ de 2005 a 2007. Escola Anna Nery Revista de Enfermagem, v. 12, n. 2 , p. 316-322, 2008.

FREITAS JÚNIOR, O. G.; CARVALHO, V. D. H.; BARROS, P. A. M.; BRAGA, M. M. Uma arquitetura para Sistemas de Gestão do Conhecimento orientada a grupos de pesquisa e desenvolvimento. Perspectivas em Gestão \& Conhecimento, v. 7, n. esp., p. 126-144, 2017.

GAO, W.; HE, X.; WANG, $H$. The impacto of knowledge integration on firm performance. Journal of International Technology and Information Management, v. 18, n. 2, p. 239, 2009.

GIL, A. C. Como elaborar projetos de pesquisa. 4. ed. São Paulo: Atlas, 2002.

GONG, Y.; KIM, T. Y.; ZHU, J.; LEE, D. R. A multilevel model of team goal orientation, information exchange, and creativity. Academy of Management Journal, v. 56, p. 827-851, 2013.

HAIR, J. F., BLACK, W. C., BABIN, B. J., ANDERSON, R. E., \& TATHAM, R. L. Análise multivariada de dados. Bookman, 2009.

HASS, M. R.; HANSEN, M. T. Different knowledge, different benefits: toward a productivity perspective on knowledge sharing in organizations. Strategic Management Journal, v. 28, n. 11, p. 1133-1153, 2007.

HENDRIKS, P.. Why share knowledge? The influence of ICT on the motivation for knowledge sharing. Knowledge and Process Management, v. 6, n. 2, p. 91, 1999.

HUANG, X.; HSIEH, J. J. P.A.; HE, W. Expertise Dissimilarity and Creativity: The Contingent Roles of Tacit and Explicit Knowledge Sharing. Journal of Applied Psychology, v. 99, n. 5, p. 816-830, 2014.

HUANG, Y. C.; WU, Y. C. J. Intellectual capital and knowledge productivity: the Taiwan biotech industry. Management Decision, v. 48, n. 4, p. 580-599, 2010.

KIM, S. L.; YUM, S. The Effect of Coworker Knowledge Sharing on Performance and Its Boundary Conditions: An Interactional Perspective. Journal of Applied Psychology, v. 100, n. 2, p.575-582, 2015.

LAKATOS, E. M.; MARCONI, M. A. Metodologia científica. 6. ed. São Paulo: Atlas, 2011.

LAWSON, B.; PETERSON, K. J.; COUSINS, P. D.; HANDFIELD, R. B. Knowledge sharing in interorganizational product development teams: the effect of formal and informal socialization mechanisms. Journal of Product Innovation Management, v. 26, n. 2, p.156-172, 2009.

LEANA, C. R.; PIL, F.K. Social capital and organizational performance: evidence from Urban 
Public Schools. Organization Science, v.17, p. 353-368, 2006.

LI, M.; GAO, F.; Why Nonaka highlights tacit knowledge: a critical review. Journal of Knowledge Management, v. 7, n. 4, pp. 6-14, 2003.

MACEDO, V.; SANTOS, N. M. B. F.; JOÃO, B. N.; SAITO, A. Tipologia do trabalhador do conhecimento: papéis e processos. Perspectivas em Gestão \& Conhecimento, v. 7, n. esp., p. 94-108, 2017.

MACHLUP, F. Semantic Quirks in Sduties of Information. In: The Sudyof Information, MACHULUP, F.; MANSFIELD, U. (org). Nova York: John Wiley \& Sons, p. 641-671, 1983.

MORIN, E. O método: o conhecimento do conhecimento. Trad. Juremir Machado da Silva. Porto Alegre: Sulina, 1986.

MOTTA, M. E. V. Capacidade de conversão do conhecimento, inovação tecnológica e o desempenho das IES do sul do Brasil. Tese de Doutorado. Pontifícia Universidade Católica/Universidade de Caxias do Sul. 2013.

NONAKA, I. A empresa criadora do conhecimento. In: Aprendizado organizacional: gestão de pessoas para a inovação contínua. HOWARD, R. Rio de Janeiro: Campus, 2000.

NONAKA, I.; TAKEUCHI, H. Criação de conhecimento na empresa: como as empresas japonesas geram a dinâmica da inovação. Rio de Janeiro: Campus, 1997.

NONAKA, I.; TOYAMA, R.; HIRATA, T. Managing Flow: teoria e casos de empresas baseadas no conhecimento. Porto Alegre: Bookman, 2011.

O'DELL, C.; GRAYSON, C. J. If only we knew what we know: identification and transfer of internal best practices. California Management Review, v. 40, p. 154-174, 1998.

PESTANA, M. H.; GAGEIRO, J. N. Análise de dados para ciências sociais: a complementariedade do SPSS. 4. ed. Lisboa: Sílabo Ltda., 2005.

PMBOK. Um guia do conjunto de conhecimentos em gerenciamentos de projetos: Guia Pmbok $^{\circledR} .4$ ed. Pensilvânia: Four Campus Boulevard, 2008.

POLANYI, M. The tacit dimension. Gloucester: Peter Smith, 1983.

POPADIUK, S.; SANTOS, A. E. M. Conhecimentos tácito explicito e cultural no planejamento da demanda JISTEM. Journal of Information Systems and Technology Management, v. 7. $\mathrm{n}$. 1, p. 205-226, 2010.

POPPER, K. R. A lógica da pesquisa científica. 10. ed. São Paulo: Cultrix, 2003.

RABELO, R. A.; FERENHOF, H. A.; RADOS, G. V.; SELIG, P. M. Gestão do conhecimento em processos de transformação organizacional: o desenvolvimento da intimidade como um fator facilitador. Perspectivas em Gestão \& Conhecimento, v. 2, n. 1, p. 21-35, 2012.

ROCHA, I. Gestão de organizações de conhecimento. Brasília: FUNADESP, 2004.

Perspectivas em Gestão \& Conhecimento, João Pessoa, v. 8, n. 2, p. 107-125, mai./ago. 2018. 
ROSSATTO, M. A. Gestão do Conhecimento: a busca da humanização, transparência, socialização e valorização do intangível. Rio de Janeiro: Interciência, 2003.

RYU, S.; HEEHO, S.; HAN, I. Knowledge sharing behavior of physicians in hospital. Expert System with Application, v. 25, p. 113-122, 2003.

SANTOS, M. J.; PAULA, C. P. A. Gestão do conhecimento no contexto da gestão escolar: estudo de caso de uma escola pública. Perspectivas em Gestão \& Conhecimento, v. 2. n. esp., p. 159174, 2012.

SOUSA, A. J. F. P.; AMARAL, S. A. Impacto do compartilhamento da informação e do conhecimento para o desenvolvimento de inovações em grandes organizações. Perspectiva em Gestão \& Conhecimento, v. 2, n. esp. P. 12-26, 2012.

SOUTO, L. F. Gestão da informação e do conhecimento: prática e reflexões. 1. ed. Rio de Janeiro: Interciência, 2014.

SPENDER, J.C. Making Knowledge the Basis of a Dynamic Theory of the Firm. Strategic Management Journal, v. 17, Special Issue: Knowledge and the Firm, p. 45-62, 1996.

STEWART, T. A. Capital intelectual: a nova vantagem competitiva das empresas. 3 ed. Rio de Janeiro: Campus, 1998.

STEWART, T. A. Compartilhamento de conhecimento. Behaviour and Information Technology, v.31, n. 11, p.1055-1056, 2012.

STRAUHS, F. R. et al. Gestão do Conhecimento nas organizações. Curitiba: Aymará Educação, 2012.

STHYHRE, A. The knowledge-intensive company and the economy of sharing: rethinking utility and knowledge management. Knowledge and Process Management, v. 9, n. 4, p. 228-236, 2002.

TURBAN, E. et al. Tecnologia da informação para gestão: transformando os negócios na economia digital. 6. ed. Porto Alegre: Bookman, 2010.

VAN DEN HOOF, B. J.; VIJVERS, J.; DE RIDDER, J. A. Knowing what to manage: foundations and applications of a knowledge-management scan. Journal of American Academy of Business, v. 21, p. 2, 2003.

WANG, N.; LIANG, H.; ZHONG, W.; XUE, Y.; XIAO, J. Resource structuring or capability building? $\mathrm{Na}$ empirical study of strategic value of information technology. Journal of Management Informational Systems, v. 29, n. 2, p. 325-367, 2012.

WANG, S.; WANG, N. X. Knowledge sharing, innovation and firm performance. Expert Systems with Applications, v. 39, n. 10, p. 8899-8908, 2012.

WANG, Z.; WANG, N.; LIANG, H. Knowledge sharing, intelectual capital and firm performance. Management Decision, v. 52, n. 2, p. 230-258, 2014.

Perspectivas em Gestão \& Conhecimento, João Pessoa, v. 8, n. 2, p. 107-125, mai./ago. 2018. 
WEGGEMAN, M. Knowledge Management: practice. Schiedam: Scriptum Management, 2000.

WILLEM, A.; SCARBROUGH, H. Social capital and political bias in knowledge sharing: an exploratory study. Human Relations, v. 59, p.1343-1370, 2006.

YAM, R. C. M.; CHAN, C. Knowledge sharing, commitment and opportunism in new product development. International Journal of Operations \& Production Management, v. 35, n. 7, p. 1056-1074, 2015.

YANG, J. T. Antecedents and consequences of knowledge sharing in international tourist hotels. International Journal of Hospitality Management, v. 29, n.1, p. 42-52, 2010.

YEON, K.; WONG, S. F.; CHANG, Y.; PARK, M.C. Knowledge sharing behavior among community members in professional research information centers. Information Development, p. 1-18, 2015.

ZHENG, M.; BAO, G. Na empirical study on knowledge sharing, affective commitment, perceived task interdependence and job involvement in chinese accounting firms. Technology Management for the Global Future, v. 3, p. $1307-1315,2006$.

Artigo recebido em 25/04/2017 e aceito para publicação em 02/07/2018 